(Supporting Information)

\title{
Chemical Constituents from the Peels of Citrus sudachi
}

Hiroyuki Nakagawa, Yoshihisa Takaishi, Naonobu Tanaka, Koichiro Tsuchiya, Hirofumi Shibata, Tomihiko Higuti.

Graduate school of Pharmaceutical Sciences, University of Tokushima, Shomachi, 1-78, Tokushima 770-8505, Japan

*To whom correspondence should be addressed.

Tel: 81-886337275.

Fax: 81-886339501.

E-mail: takaishi@ph.tokushima-u.ac.jp.

University of Tokushima. 


\section{Known Compounds from the Peels of Citrus sudachi}

Following 27 known compounds were isolated from the peels of C. sudachi; isoobacunoic acid (8), ${ }^{1}$ limonin $(\mathbf{9}),{ }^{2}$ methyl deacetylnomilinate $(\mathbf{1 0}),{ }^{3}$ nomilinic acid (11), ${ }^{4}$ vanillic acid (12), ${ }^{5} 1 S, 2 S, 4 R$-limonene-1,2-diol (13), ${ }^{6}(+)-4 S$-7-hydroxypiperitone (14), ${ }^{7}$ methyl ferulate $(\mathbf{1 5}),{ }^{8}$ ferulic acid (16),${ }^{9}$ citrusin III $(\mathbf{1 7}),{ }^{10}$ citrusin IX $(\mathbf{1 8}),{ }^{11}$ sudachitin (6), ${ }^{12} 3^{1}$-demethoxysudachitin (7), ${ }^{12}$ 7-methylsudachitin (19), ${ }^{13}$ xanthomicrol (20), ${ }^{13}$ jaceosidin $(\mathbf{2 1}),{ }^{14}$ sudachiin B $(\mathbf{2 2}),{ }^{15}$ sudachiin C (23) $){ }^{15}$ prunin $(\mathbf{2 4}),{ }^{16}$ narirutin (25), ${ }^{17}$ naringin $(\mathbf{2 6}),{ }^{17}$ hesperidin $(\mathbf{2 7}),{ }^{17}$ neohesperidin $(\mathbf{2 8}),{ }^{18}$ eriocitrin $(\mathbf{2 9}),{ }^{19}$ poncirin (30), ${ }^{20}$ hesperetin 7-O-(2",6"-di- $O$ - $\alpha$-rhamnopyranosyl)- $\beta$-glucopyranoside $(\mathbf{3 1}),{ }^{17}$ naringenin 7-O-(2",6"-di- $O$ - $\alpha$-rhamnopyranosyl)- $\beta$-glucopyranoside $(\mathbf{3 2}) .^{21} \quad$ Their structure were identified by the analysis of their NMR spectral data, and then by comparison with those of literature listed below, respectively. Eleven compounds $(\mathbf{8}, \mathbf{1 0}$, $12-18,24,32)$ were the first isolation from C. sudachi.

\section{Isolation of Known Compounds}

The EtOAc soluble fraction ( $54 \mathrm{~g}$, the extraction and the partion were described in text) was subjected to silica gel column chromatography (1 kg, 11 x $100 \mathrm{~cm})$. The column was eluted with solvents of increasing polarity ( $n$-hexane-EtOAc, EtOAc, EtOAc-MeOH, MeOH) to give 15 major fractions (frs. 1-15). Fraction 1 (460 mg) was separated on GPC $\left(\mathrm{CHCl}_{3}\right)$, Si HPLC $\left(\mathrm{CHCl}_{3}-\mathrm{MeOH}\right.$, 95:5) to give 15 (5 mg). Fraction 3 (477 mg) was separated by Si HPLC $\left(\mathrm{CHCl}_{3}-\mathrm{MeOH}\right), \mathrm{GPC}(\mathrm{MeOH})$ to give 12 (3 $\mathrm{mg}), \mathbf{1 3}(15 \mathrm{mg}), \mathbf{1 9}(6 \mathrm{mg}), \mathbf{2 0}(10 \mathrm{mg})$. Fraction 4 (3.7 g) was chromatographed on a silica gel column $\left(\mathrm{CHCl}_{3}-\mathrm{MeOH}\right)$ to give three fractions (frs 2.1-2.3) and 7 (300 mg). Fraction 2.3 was separated by a Toyopearl HW-40 column $\left(\mathrm{CHCl}_{3}-\mathrm{MeOH}, 1: 1\right)$, Si 
HPLC $\left(\mathrm{CHCl}_{3}-\mathrm{MeOH}\right), \mathrm{GPC}(\mathrm{MeOH})$ to give $14(7 \mathrm{mg}), \mathbf{1 6}(8 \mathrm{mg}), 21(5 \mathrm{mg})$. Fraction $5(1.1 \mathrm{~g})$ was subjected on Si HPLC $\left(\mathrm{CHCl}_{3}-\mathrm{MeOH}\right)$ to give 6 (360 mg). Fraction 6 (2.4 g) was chromatographed on a Toyopearl HW-40 column $\left(\mathrm{CHCl}_{3}-\mathrm{MeOH}\right)$ to give four fractions (frs 6.1-6.4). Fraction 6.3 was separated by Si HPLC $\left(\mathrm{CHCl}_{3}-\mathrm{MeOH}\right), \mathrm{GPC}$ $(\mathrm{MeOH})$ to give 8 (13 mg), 10 (44 mg). Fraction 7 (19.0 g) was chromatographed on a silica gel column $\left(\mathrm{CHCl}_{3}-\mathrm{MeOH}\right)$ to give seven fractions (frs 7.1-7.7). Fraction 7.3 was subjected on recrystallization $(\mathrm{MeOH})$; fraction 7.4 subjected on Si HPLC (hexaneEtOAc) to give $9(1.0 \mathrm{~g}), 11(5 \mathrm{mg})$, respectively. Fraction $11(3.0 \mathrm{~g})$ was separated by a Sephadex LH-20 $(\mathrm{MeOH})$ column, GPC $(\mathrm{MeOH})$, ODS $\left(\mathrm{MeOH}-\mathrm{H}_{2} \mathrm{O}\right)$ to give 24 (2 $\mathrm{mg})$. Fractions $12(1.9 \mathrm{~g})$ was recrystallized from $\mathrm{MeOH}$ to give 22 (144 mg). Fraction 13 (1.9 g) was separated by a Sephadex LH-20 (MeOH) column, recrystallization $(\mathrm{MeOH}), \mathrm{GPC}(\mathrm{MeOH})$ to give $25(138 \mathrm{mg}), 29(60 \mathrm{mg})$. Fraction $14(8.3 \mathrm{~g})$ was separated by a Sephadex LH-20 $(\mathrm{MeOH})$ column to give seven fractions (frs 14.1-14.7) and a mixture of 27, 28 (2.5 g, 3:2). Fraction 14.7 was separated by GPC (MeOH), ODS $\left(\mathrm{MeOH}-\mathrm{H}_{2} \mathrm{O}\right)$ to give $27(193 \mathrm{mg}), \mathbf{3 0}(1 \mathrm{mg})$, a mixture of 25, $26(85 \mathrm{mg}, 1: 1)$ and that of 26, 29 (63 mg, 2:5). Fraction 15 (1.5 g) was separated by a Sephadex LH-20 (MeOH) column, GPC (MeOH), ODS $\left(\mathrm{MeOH}-\mathrm{H}_{2} \mathrm{O}\right)$ to give $17(63 \mathrm{mg}), 18(29 \mathrm{mg}), 23$ (13 mg), $31(11 \mathrm{mg})$.

One percent of $n-\mathrm{BuOH}$ soluble fraction (3.8 g) was chromatographed on a Sephadex LH-20 (MeOH) column chromatography to give five fractions (frs 1-5). Fraction 2 (1.3 g) was subjected on GPC (MeOH) to give 31 (4 mg), $32(21 \mathrm{mg})$. Fraction 3 (460 mg) was separated by GPC $(\mathrm{MeOH}), \mathrm{ODS}\left(\mathrm{MeOH}-\mathrm{H}_{2} \mathrm{O}\right)$ to give a mixture of $\mathbf{2 5}, \mathbf{2 6}(104$ $\mathrm{mg}, 1: 1)$ and that of 27, 28 (106 $\mathrm{mg}, 1: 2)$. Fractions 4 (155 mg) was subjected on GPC 
$(\mathrm{MeOH})$ to give a mixture of $\mathbf{2 5}, \mathbf{2 6}(36 \mathrm{mg}, 3: 2)$ and that of $\mathbf{2 7}, \mathbf{2 8}(22 \mathrm{mg}, 1: 1)$, that of 26, 29 (37 mg, 1:3).

\section{References and Notes}

[1] Raymond, D.B.; Hasegawa, S.; Zareb, H. Phytochemistry 1989, 28, 2777-2781.

[2] Ming, K.; Gray A.I.; Waterman, P.G. Journal of Natural Products 1987, 50, 11601163.

[3] Bennet, R.D.; Hasegawa, S. Tetrahedron 1981, 37, 17-24.

[4] Ellis, B.E.; Amrhein, N. Phytochemistry 1971, 10, 3069-3072.

[5] Sakushima, A.; Coskun, M.; Maoka, T. Phytochemistry 1995, 40, 257-261..

[6] Demyttenaere, J.C.R.; Belleghem, K.V.; Kimpe, N.D. Phytochemistry 2001, 57, 199208.

[7] Delgado, G.; Rios, M.Y. Phytochemistry 1991, 30, 3129-3131.

[8] Fujita, M.; Inoue, T; Nagai, M. Yakugaku zasshi 1985, 105, 240-248.

[9] Ternai, B.; Markham, K.R. Tetrahedron 1976, 32, 565-569.

[10] Matsubara, Y.; Yusa, T.; Sawabe, A.; Iizuka, Y.; Takekuma, S.; Yoshida, Y. Agric. Biol. Chem. 1991, 55, 2923-2929.

[11] Matsumoto, T.; Shishido, A.; Takeya, K. Tennen Yuki Kagobutsu Toronkai Koen Yoshishiu 2001, 43th, 407-412.

[12] Greenham, J.; Vassiliades, D.D.; Harborne, J.B.; Williams, C.A.; Eagles, J.; Grayer, R.J.; Veitch, N.C. Phytochemistry 2001, 56, 87-91.

[13] Horie, T; Nakayama, M. Phytochemistry 1981, 20, 337-338.

[14] Martinez, V.; Barbera, O.; Parareda, J.S.; Marco, J.A. Phytochemistry 1987, 26, 
2619-2624.

[15] Horie, T.; Tsukayama, M.; Yamada, T.; Miura, I.; Nakayama, M. Phytochemistry 1986, 25, 2621-2624.

[16] Lewinsohn, E.; Berman, E.; Mazur, Y.; Gressel, J. Phytochemistry 1986, 25, 25312535.

[17] Kumamoto, H.; Matsubara, Y.; Iizuka, Y.; Okamoto, K.; Yokoi, K. Nippon Nogeikagaku Kaishi 1985, 59, 683-687.

[18] Matsubara, Y.; Kumamoto, H.; Yonemoto, H.; Iizuka, Y.; Okamoto, K.; Yokoi, K. Nippon Nogeikagaku Kaishi 1985, 59, 405-410.

[19] Miyake, Y.; Yamamoto, K.; Osawa, T. Food Sci. Technol. Int. Tokyo 1997, 3, 8489.

[20] Kim, D.H.; Bae, E.A.; Han, M.J. Biol. Pharm. Bull. 1999, 22, 422-424.

[21] Kim, H.K.; Jeon, W.K.; Ko, B.S. Planta Medica 2001, 67, 548-549. 